\title{
Expression pattern of annelid Zic in embryonic development of the oligochaete Tubifex tubifex
}

\author{
Hirokazu Takahashi • Takashi Shimizu • Jun Aruga
}

Received: 12 June 2008 / Accepted: 5 September 2008 / Published online: 23 September 2008

(C) Springer-Verlag 2008

\begin{abstract}
Embryonic expression of a Zic homologue $($ Ttu-Zic) was examined in the oligochaete annelid Tubifex tubifex. The body plan of T. tubifex is characterized by obvious segmentation in the ectoderm and mesoderm. Ttu-Zic expression is detected in the mesodermal germ band and a subset of micromere descendants. Ttu-Zic is transiently expressed in primary $\mathrm{m}$-blast cells (i.e., founder cells of mesodermal segments) as early as the time of their birth from M teloblasts. During its development, each mesodermal segment experiences two additional phases of Ttu-Zic expression. Ttu-Zic expression in micromere descendants is seen on the anterior surfaces of embryos undergoing teloblastogenesis; subsequently, these cells proliferate to form bilateral clusters, which then become internalized. Finally, clusters of Ttu-Zic-expressing cells are found in the center of the prostomium, corresponding to the cerebral ganglion. The Ttu-Zic expression profile in the early embryogenesis of $T$. tubifex may be homologous to those of evolutionarily distant animals.
\end{abstract}

Communicated by D.A. Weisblat

H. Takahashi $\cdot$ J. Aruga $(\square)$

Laboratory for Behavioral and Developmental Disorders

(formerly Laboratory for Comparative Neurogenesis),

RIKEN Brain Science Institute,

Wako 351-0198, Japan

e-mail: jaruga@brain.riken.jp

T. Shimizu

Graduate School of Science, Hokkaido University,

Sapporo 060-0810, Japan
Keywords Oligochaete annelid - Tubifex tubifex .

Body plan · Mesodermal Development .

Neural development $\cdot$ Transcription factor $\cdot$ Zic $\cdot$ Msx $\cdot$ Emx

\section{Introduction}

Recent molecular phylogenetic analyses have revealed that Zic family genes are found in a wide range of eumetazoans (Aruga et al. 2006, 2007). Expression and functional analyses indicate that Zic zinc finger proteins commonly play roles in cell differentiation and proliferation in the early embryonic stages in Deuterostomia, Ecdysozoa, and Cnidaria (reviewed in Aruga 2004; Merzdorf 2007). However, an obvious defect in our understanding of the Zic phylogeny is the lack of information on lophotrochozoan Zic in the literature, except in terms of gene structure. Lophotrochozoa is the sister taxon of Ecdysozoa in the Protostomia and includes mollusks, annelids, and platyhelminthes.

As a model lophotrochozoan animal, the oligochaete annelid Tubifex tubifex, a freshwater sludgeworm, has a body organization that is notable for its clear segmentation (Shimizu 1982; Goto et al. 1999a; Nakamoto et al. 2000). The ectodermal and mesodermal segmental structures arise from five bilateral pairs of coherent longitudinal columns (bandlets) of blast cells that are formed by five bilateral pairs of embryonic stem cells called teloblasts (named $\mathrm{M}$, $\mathrm{N}, \mathrm{O}, \mathrm{P}$, and $\mathrm{Q}$ ). The teloblasts are located at the posterior end of the embryo and generate daughter cells one by one through asymmetrical cell divisions. The older daughter cells are located in the anterior side of each bandlet, making it easy for researchers to observe the series of differentiation 
processes in the array of body segments. Among the teloblasts, the $\mathrm{M}$ teloblast lineage contributes solely to the oligocheates' mesodermal segments (Goto et al. 1999b; Nakamoto et al. 2000).

In this study, we examined the expression of Tubifex Zic (Ttu-Zic) in the course of embryonic development to better understand the evolution of Zic gene expression. The results revealed that some expression features are shared with other bilaterian Zic homologues.

\section{Materials and methods}

Animals

T. tubifex were obtained as described (Shimizu 1982). For experiments, embryos were freed from cocoons in the culture medium (Shimizu 1982).

PCR cloning and RT-PCR analysis

RNAs were isolated by using TRIzol reagent (Invitrogen, Carlsbad, CA, USA) in accordance with the manufacturer's recommendations. Complementary DNAs (cDNAs) were generated by reverse transcription using PrimeScript reverse transcriptase (Takara Bio, Shiga, Japan). The following primers were used for the reverse transcription polymerase chain reaction (RT-PCR) analysis: Emx-F, 5'-GTTTCC AGGTCTGACGGATT-3'; Emx-R, 5'-GGTGTCGATTCC AATGCAGT-3'; Msx-F，5'-GTCATGTCTGGTTCTTCG AC-3'; Msx-R, 5'-GCCTAGAATACCGTCAACTG-3'; ZicF, 5'-CGTACAACTGCAAGATGCGA-3'; Zic-R, 5'TGTCGTCTGAAACCGAGAAC-3'; Actin-F, 5'-TGGGAY GAYATGGARAARAT; Actin-R, 5'-GCCATYTCYTGYT CRAA-3'. The PCR reaction was carried out with ExTaq DNA polymerase (Takara Bio) in the presence of BD TaqStart anti-Taq antibody (BD Biosciences, San Jose, CA, USA). Each PCR consisted of 30 cycles of $94^{\circ} \mathrm{C}$ for $1 \mathrm{~min}, 58^{\circ} \mathrm{C}$ for $1 \mathrm{~min}$, and $72^{\circ} \mathrm{C}$ for $2 \mathrm{~min}$. Tubifex Emx cDNA was obtained by a PCR screening of Tubifex homeodomain-containing genes (unpublished results). The Emx sequence was deposited in the DDBJ database with accession number AB439506. Tubifex Zic (Ttu-Zic, AB231870) and Tubifex Msx (Ttu-Msx, AB302957) cDNAs were previously described (Aruga et al. 2006; Takahashi et al. 2008).

In situ hybridization

In situ hybridization was performed as described (Matsuo and Shimizu 2006). The stained embryos were cleared by placing them in a 1:2 mixture of benzyl alcohol and benzyl benzoate.

\section{Results and discussion}

Comparison of temporal expression profiles

The processes of Tubifex development have been described in previous studies (Shimizu 1982; Goto et al. 1999a; Nakamoto et al. 2000). Figure 1a summarizes the Tubifex developmental processes relevant to this study; we used this figure as a staging diagram. The temporal expression profile was first examined by RT-PCR analysis (Fig. 1b) using the RNAs from representative developmental stages

A
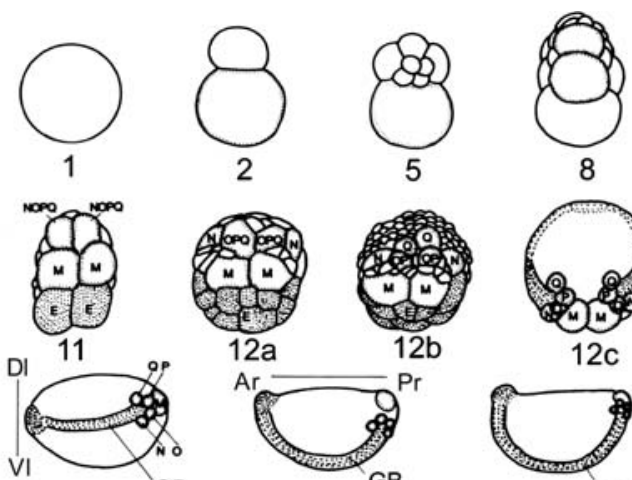

$\mathrm{Pr}$

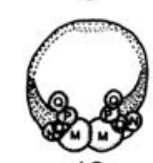

$12 \mathrm{c}$
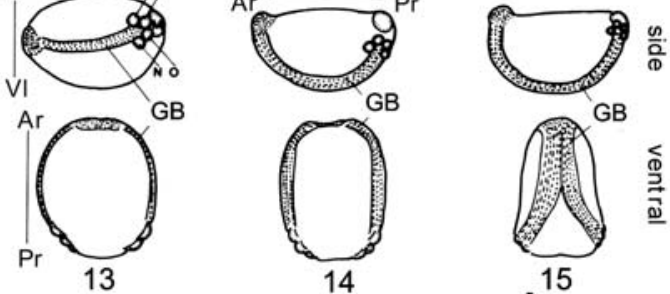

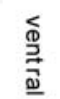
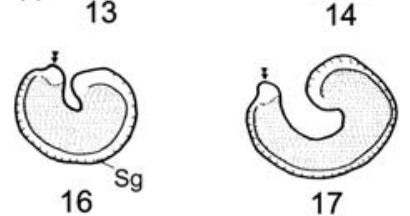

17

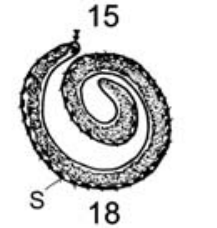

B

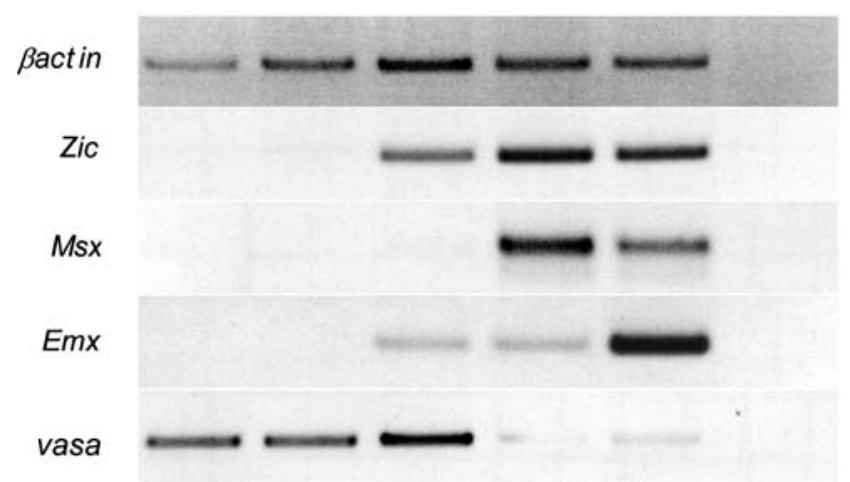

Fig. 1 a Summary of Tubifex development (for details, see Shimizu 1982). $A r$ Anterior, $D l$ dorsal, $E$ endodermal cells, $G B$ germ band, $P r$ posterior, $S$ setae, $S g$ segment, $V l$ ventral, double arrowheads prostomium (which includes cerebral ganglion). $M, N, O, P$, and $Q$ indicate teloblasts, and $N O P Q$ and $O P Q$ indicate their precursors. b Temporal expression profiles of Zic and some representative molecular markers in the course of Tubifex embryonic development, as determined by RT-PCR analysis. $-R T$ indicates a control PCR using the stage 15 RNA without reverse transcription 

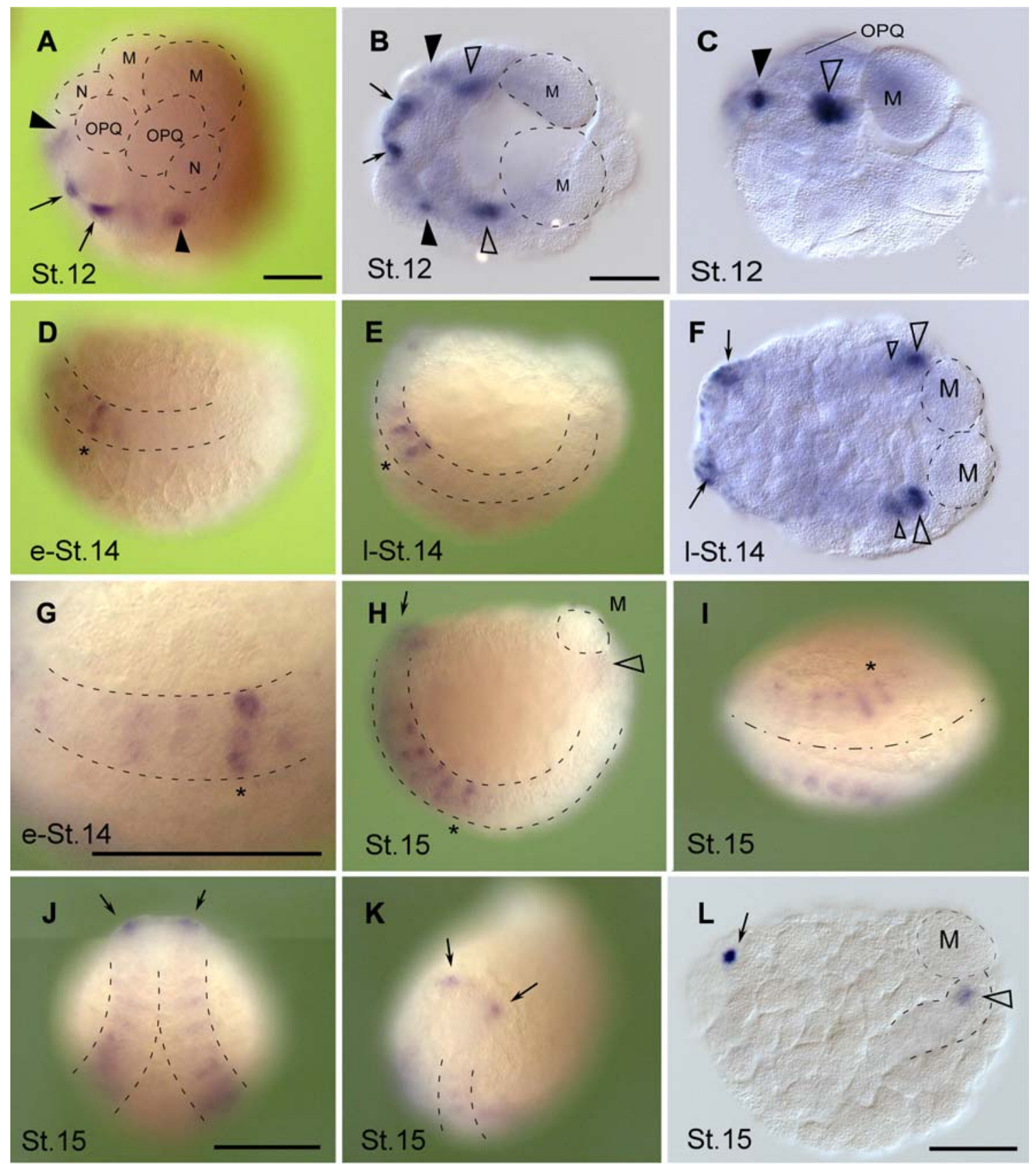

Fig. $2 T t u$-Zic expression in early embryogenesis. a Antero-dorsal view of a stage 12 embryo shortly after division of NOPQ into N and OPQ. Uncleared specimen. Arrows indicate a pair of anteriorly located surface cells. Solid arrowheads indicate stained cells located internally. b, c Cleared specimens of stage 12 embryos (anterior to left). b Dorsal view. In this optical section, three kinds of stained cells are seen: anteriorly located surface cells (arrows), primary m-blast cells (open arrowheads) in front of $\mathrm{M}$ teloblasts, and anteriorly located inner cells (solid arrowheads). c Lateral view (dorsal at top). Open arrowhead indicates a primary m-blast cell; solid arrowhead indicates an anteriorly located inner cell. $\mathbf{d}-\mathbf{f}$ Early $(\mathbf{d}, \mathbf{g})$ and late $(\mathbf{e}, \mathbf{f})$ stage 14 embryos. d, e Left side views with anterior to the left and dorsal to the top. Asterisks indicate positions of the posterior-most dumbbell- shaped structures. f Dorsal view with anterior to the left. Cleared specimen. Note two stained cells (open arrowheads) in front of $\mathrm{M}$ teloblasts. Arrows indicate anteriorly located clusters of stained cells. g Enlargement of $\mathbf{d}$ showing transverse dumbbell-shaped structures in the mesodermal germ band. h-I Stage 15 embryos viewed from the left side (h, l), ventral (i), anterior (j), and antero-dorsal (k). Mesodermal germ bands are demarcated by broken lines. Circles of broken lines in $\mathbf{f}, \mathbf{h}$, and $\mathbf{I}$ indicate $\mathbf{M}$ teloblasts. Dotted line in $\mathbf{i}$ indicates the ventral midline. I Cleared specimen (anterior to left). Arrow indicates an anteriorly located dot of stained cells; open arrowhead indicates a primary $\mathrm{m}$-blast cell with hybridization signals. Scale bar, $100 \mu \mathrm{m}$. Bars in $\mathbf{a}$ and $\mathbf{b}$ are common to $\mathbf{d}, \mathbf{e}, \mathbf{h}, \mathbf{i}, \mathbf{k}$ and $\mathbf{c}, \mathbf{f}$, respectively 
(stages 1, 2-8, 12, 15, and 16-17). In the course of embryonic development, Ttu-Zic expression was first detected at the beginning of teloblastogenesis (stage 12), peaked at the beginning of germ band coalescence (stage 15), and continued thereafter (stages 16-17). Ttu-Emx showed strong expression at stages 16 and 17 and weak expression at stages 12 and 15. Ttu-Msx expression was first detected at stage 15 and continued further. Tubifex vasa showed an earlier expression peak, in agreement with the results of a previous study (Oyama and Shimizu 2007), thus validating our results.

\section{Expression of Ttu-Zic in early embryogenesis}

We performed in situ hybridization analyses using a Ttu-Zic antisense riboprobe and a series of embryos from the onecell stage (stage 1) to the vermicular form stage (stage 18). In embryos processed for in situ hybridization with a sense probe, no staining was detected from stage 1 to stage 18 (data not shown).

In the course of Tubifex development, marked expression of Ttu-Zic was first detected at the transition to stage 12, when a proteloblast, NOPQ, on each side of the embryo divides into $\mathrm{N}$ and OPQ (Fig. 1). At this stage, the signals can be seen as two bilateral pairs of dots in uncleared embryos (Fig. 2a). One pair is located on the anterior surface of the micromere cap comprising the micromere progeny. The Ttu-Zic-positive cells in the micromere cap appear to be connected to each other by thin processes, which assume an arched shape (Fig. 2a, arrows; Fig. 2b). In contrast, the other pairs of Ttu-Zic-positive cells are not surface cells and are located in the interior region of the embryo (Fig. 2a, solid arrowheads); the embryonic origin of these interior cells remains to be explored. As indicated by the open arrowheads in Fig. 2b, c, cleared specimens of the same stage exhibit additional stained cells, which are located in front of $\mathrm{M}$ teloblasts (Fig. 2b, c, open arrowheads). Judging from their size and position, it is safe to say that these positive cells correspond to the primary m-blast cells that have most recently been formed from the $M$ teloblasts. Given the absence of Ttu-Zic expression in the M teloblasts, it is likely that nascent $m$-blast cells initiate zygotic transcription of this gene upon their birth.

Expression of Ttu-Zic during germ band elongation (stages 14 and 15)

In embryos undergoing gastrulation (stages 13 to 16), germ bands elongate, curve round toward the ventral midline, and coalesce with each other along the ventral midline (Fig. 1, stages 13-15); this coalescence begins at the anterior end of the embryo and proceeds in an anterior-to-posterior fashion.

We hybridized stage 14 embryos with the antisense TtuZic riboprobe (Fig. 2d-f, 4a). Ttu-Zic is expressed in the
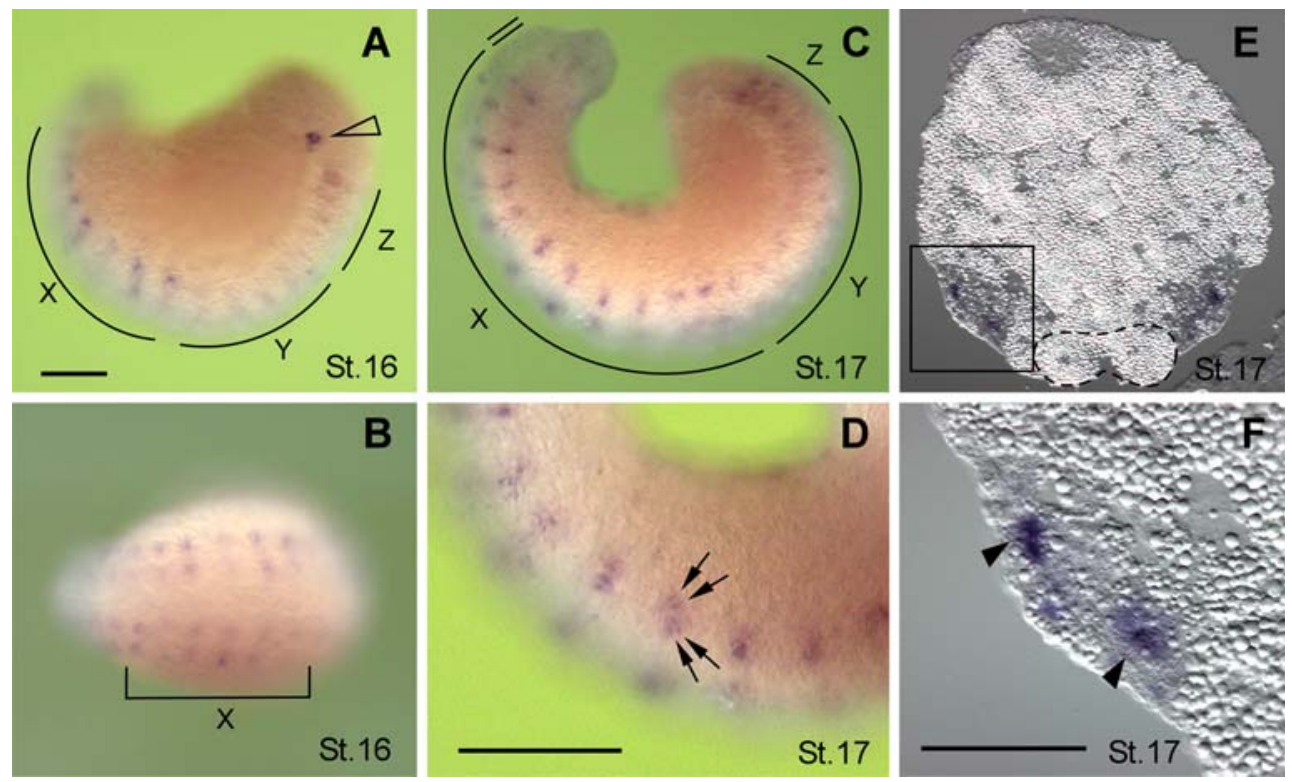

Fig. 3 Ttu-Zic expression during embryo body elongation. a, b Stage 16 embryo viewed from the left (a) and antero-ventral (b) sides. Anterior is to the left. c, d Stage 17 embryo viewed from the left. Anterior is to the left. c Parallel line running along the ventral surface indicates the position of the first segment. Note the absence of paired dots in this segment. In a-c, curved lines and a bracket labeled with $X$ to $Z$ indicate the three domains along the mesodermal germ band. d
Enlargement of $\mathbf{c}$ showing the anterior half of the embryo. Note stained conical structures (arrows). e, f Transverse section of the third segment of a stage 17 embryo. Dorsal is to the top. Broken line demarcates a ventral ganglion. f An enlargement of the lower left of $\mathbf{e}$ showing stained, paired dots (arrowheads). Scale bar, $100 \mu \mathrm{m}$. Scale bar in $\mathbf{a}$ is common to $\mathbf{b}, \mathbf{c}$, and $\mathbf{e}$ 
anterior-most pair of cell clusters that is first seen at stage 12 (Fig. 2f, arrows) and in the mesodermal germ bands (Fig. 2d, e). The anterior cell cluster is composed of five or six stained cells, and when viewed from the side, it appears as a discrete dot (Fig. 21, arrow; the fate of this cell cluster will be described later).

The expression of Ttu-Zic in the mesodermal germ band during stage 14 is restricted to four or five anterior-most segments and one or two posterior-most "segments" (nascent primary m-blast cells; Fig. 2f, open arrowheads). In each of the anterior segments, Ttu-Zic-expressing cells are organized in transverse dumbbell-shaped structures (Fig. 2g); the hybridization signal is apparently stronger in the most posterior segment than in the more anterior segments (Fig. 2d, e, g). As the development proceeds, Ttu-Zic-expressing cells emerge in the more posterior segments as well. At early stage 15, eight anterior segments exhibit Ttu-Zic-expressing cells on either side of the embryo (Fig. 2h, i).
Expression of Ttu-Zic during embryo body elongation (stages 16 to 18 )

In Tubifex embryos, gastrulation is followed by dorsal-ward expansion of germ bands and by simultaneous body elongation, which proceeds in an anterior-to-posterior fashion (see Fig. 1, stages 16-18). By the beginning of stage 16 (i.e., at the beginning of body elongation), the number of Ttu-Zic-positive segments has increased to 16 (Fig. 3a and 4b). On the basis of the distribution pattern of Ttu-Zic-expressing cells in individual segments, three domains are discernible along the germ band: the anterior domain (domain $\mathrm{X}$ in Fig. 3a) with paired dots on either side of each segment (Fig. 3b), the mid domain ( $Y$ in Fig. 3a) with a single dot (with weak signals) in each segment, and the posterior domain ( $Z$ in Fig. 3a) with positive cells organized in the transverse dumbbell-shaped structures seen in stage 14 embryos (see Fig. 2e, g). TtuZic-positive cells are absent in the first segment (Fig. 3a)

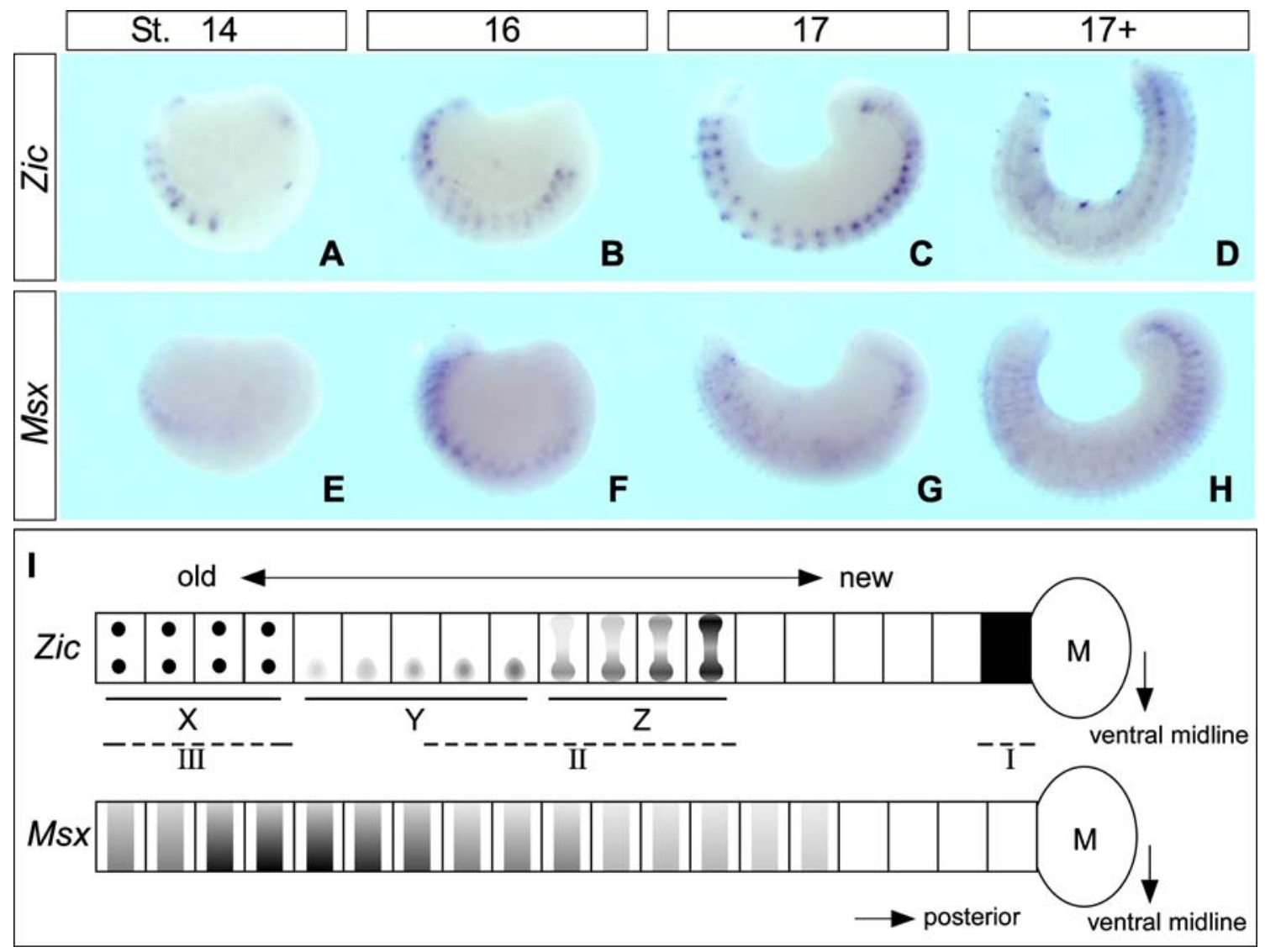

Fig. 4 Comparative analysis of Ttu-Zic and Ttu-Msx expression profiles in mesodermal lineage. Lateral views of stage $14(\mathbf{a}, \mathbf{e}), 16(\mathbf{b}$, f), 17 (c, g), and later $17(17+, \mathbf{d}, \mathbf{h})$ embryos. In all panels, anterior is to the left and dorsal is to the top. The embryos were hybridized with the Ttu-Zic (a-d) or Ttu-Msx antisense probe (e-h). i Schematic illustration of Ttu-Zic and Ttu-Msx expression in the mesodermal germ bands derived from $\mathrm{M}$ teloblasts. Each square represents a meso- dermal segment that coincides with the body segment. Anterior is to the left; dorsal is to the top. The three domains $X, Y$, and $Z$ correspond to those indicated in Fig. 3a. The antero-posterior position in the segments is arbitrarily indicated at the middle. Roman numerals represent the tentative triphasic expression pattern of Ttu-Zic in the mesodermal lineage 
and in the region behind the above-mentioned posterior domain (Z), except for a single cell (Fig. 3a, open arrowhead) located in front of the M teloblasts.

At stage 17, Ttu-Zic-expressing cells are organized in paired dots in most of the mesodermal segments, except in the posterior region of the embryo (Fig. $3 c$ and $4 c$ ), where positive cells are apparently organized in dumbbell-shaped structures (Fig. 3c, domain Z). At this stage as well, TtuZic-positive cells are not detected in the first segment (indicated by a thin line in Fig. 3c). At late stage 17, the cessation of Ttu-Zic expression begins at the anterior-most region of the embryo and proceeds in an anterior-toposterior fashion (Fig. 4d).

In summary, we suggest that the Ttu-Zic expression in mesodermal segments is a triphasic process. The first phase of expression occurs in primary m-blast cells shortly after

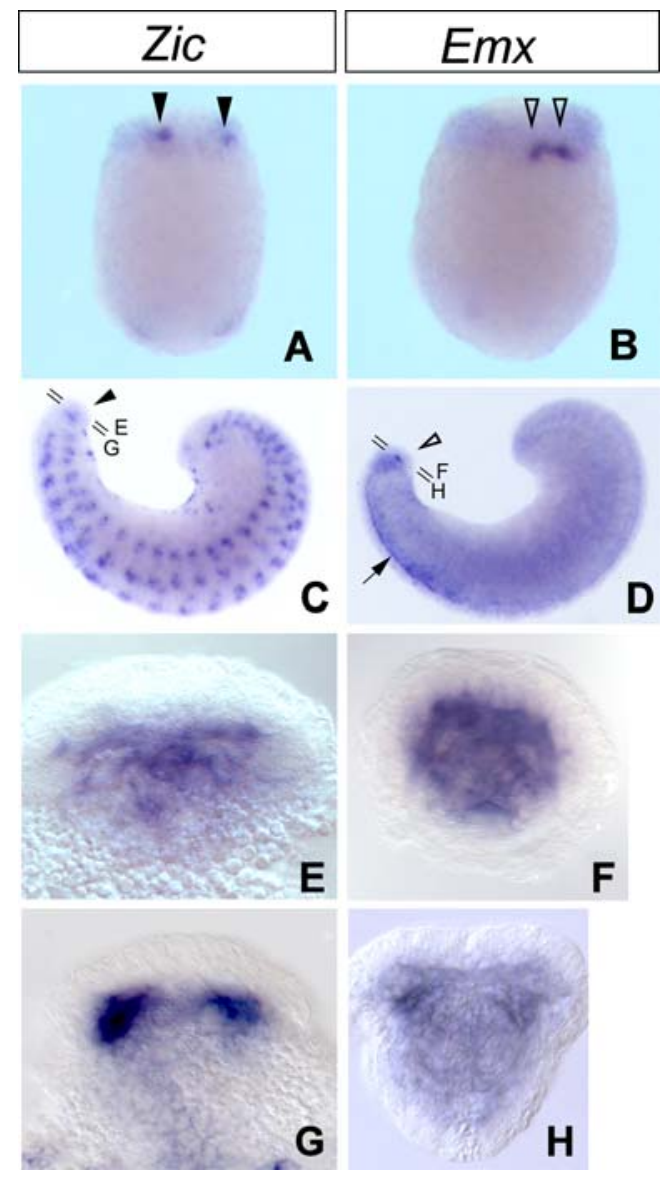

Fig. 5 Rostral expression of Ttu-Zic in the course of later embryonic development. In situ hybridization signals given by the Ttu-Zic (a, c, $\mathbf{e}, \mathbf{g})$ and $T t u-E m x$ antisense $(\mathbf{b}, \mathbf{d}, \mathbf{f}, \mathbf{h})$ probes are shown. a, b Ventral views of mid gastrula stage embryos (St. 14). c, d Lateral views of stage 17 embryos. Arrowheads indicate the rostral-end signal of each gene (Ttu-Zic, closed; Ttu-Emx, open). Arrow indicates the expression in ventral ganglia. Bars indicate the positions of transverse sections shown in $\mathbf{e}-\mathbf{h}$. $\mathbf{e}-\mathbf{h}$ Transverse sections through the prostomium and ventral first body segment of stage 17 embryos. Sections of $\mathbf{e}, \mathbf{f}$ are more anterior than those of $\mathbf{g}, \mathbf{h}$ their birth. The expression is apparently transient and ceases upon the first cell division (which takes place concurrently with the next division of their mother cell, the M teloblast; see Goto et al. 1999b). The second phase of Ttu-Zic expression commences some time after cessation of the first expression, and Ttu-Zic-expressing cells are organized in transverse dumbbell-shaped structures. These structures exhibit signals that gradually become weaker with time, and they appear to be transformed into dot-like structures with weak signals. Ttu-Zic expression resumes again sometime after the attenuation of the second phase. This third phase of expression consists of intensely stained, paired dots of Ttu-Zic-expressing cells on either side of the embryo.

The Ttu-Zic expression profiles in the mesodermal lineage may accommodate the differentiation stage of each segment. To learn whether the aforementioned spatiotemporal expression pattern of Ttu-Zic is widespread among genes expressed in the mesodermal cell lineage (i.e., in the germ band), we examined the expression pattern of another developmental tool-kit gene, Ttu-Msx (Takahashi et al. 2008), which was shown to be expressed in the mesodermal lineage in a preliminary observation. Expression of Ttu-Msx begins at late stage 14 , and hybridization signals are detected as transverse, regularly repeating stripes in the anterior region of the mesodermal germ band (Fig. 4e). As development proceeds, Ttu-Msx-positive stripes emerge in the more posterior region as well; concurrently with this, the preexisting stripes in the anterior region exhibit stronger signals than before (compare Fig. 4f with Fig. 4e). The signals in each segment become weaker during development up to stage 17 (Fig. 4g, h). As a result, the expression pattern of Ttu-Msx is distinct from that of Ttu-Zic (Fig. 4i). It is apparent that the triphasic mode of expression is a feature specific to Ttu-Zic.

\section{Ttu-Zic expression in putative setal sacs}

We next examined the identity of the paired dots in the third phase of expression. The paired dots appeared at stage 16 and later (Fig. 3a-c) and were arranged in the same transverse plane around the periphery of each segment and located in the interior region, but not on the embryo surface (Fig. 3e, f). Furthermore, each dot was composed of two pairs of signal-forming conical shapes (Fig. 3d).

Judging from the spatial pattern of their occurrence, it is likely that the paired dots correspond to the setal (chaetal) sacs that exist in every embryonically produced segment except the first. In previous studies, we have suggested that the cells comprising Tubifex setal sacs express Ttu-dpp and Ttu-p68 (a DEAD-box RNA helicase; Matsuo and Shimizu 2006; Oyama et al. 2008). Interestingly, Ttu-dpp-expressing cells are organized in dot-like structures, which are 
composed of conical units like those seen in the dots of TtuZic-expressing cells (Matsuo and Shimizu 2006). However, Ttu-Zic-expressing cells may be distinct from those expressing Ttu-dpp because Ttu-dpp-expressing cells in the setal sacs are of ectodermal origin (Matsuo and Shimizu 2006). Given that Ttu-dpp-expressing cells contribute chaetoblasts that produce setae (chaetae) for setal (chaetal) sacs, it is plausible that Ttu-Zic-expressing cells contribute mesodermal tissues such as muscle tissue to the setal sacs.

Expression of Zic in cerebral-ganglion-forming region

The anteriorly localized, bilateral clusters of Ttu-Zicexpressing cells, which are located in the outer cell layer during stages 14 and 15 (Fig. 2f, j, k, l), appear to be internalized during subsequent development. Stage 17 embryos exhibit a cluster of Ttu-Zic-expressing cells in the central region, but not the periphery, of the prostomium (Fig. 5c). Close examination of the cell cluster suggests that it could result from the apposition of separate clusters of TtuZic-expressing cells (Fig. 5e, g). From their location, it seems likely that these positive cells correspond to a subpopulation of micromere progeny that form a cerebral ganglion.

Since no reliable markers for cerebral ganglion precursors were available in T. tubifex, we cloned a Tubifex homologue of the Emx gene (Ttu-Emx) as a potential cerebral ganglion marker and then examined its expression pattern. Emx homologues are expressed in early embryonic brains in both Drosophila and the mouse (Lichtneckert and Reichert 2005).

As expected, Ttu-Emx hybridization signals were observed in the cerebral-ganglion-forming region in the late vermiform embryo (Fig. 5d, f, h); in younger embryos, Ttu$E m x$-expressing cells were found on the surface of the rostral end (Fig. 5b). The rostral Ttu-Emx signals were located near the Ttu-Zic-expressing region and partly overlapped with the Ttu-Zic-expressing region in the vermiform embryos (Fig. 5e-h). The hybridization signals were stronger in the rostral part of the Ttu-Emx-expressing region than in the caudal part (Fig. 5f, h), whereas the Ttu-Zic signals were stronger in the caudal part than in the rostral part (Fig. 5e, g). The gross localization of the Ttu-Zic hybridization signal and comparison of the localization with the Ttu-Emx expression pattern suggest that at least some of Ttu-Zic-expressing cells residing in the central region of the prostomium contribute to formation of the cerebral ganglion.

Comparison of Ttu-Zic expression profiles with those of other animals

We reported in this paper the first Zic expression profile in lophotorochozoan embryonic development. In previous studies, the expression profiles of Zic homologues in deuterostomes (vertebrates and ascidians) and ecdysozoans (flies and nematodes) have been described, and a shared expression feature in the earliest neural tissue have been pointed out (Aruga 2004). Furthermore, in Cnidaria, hydra Zic (Hyzic) is expressed just before achaete-scute homologue expression; this is similar to what occurs in vertebrate neurogenesis (Lindgens et al. 2004). Expression in the presumptive cerebral-ganglion-forming cells seems to be homologous to the expression of the Zic homologues in the earliest neuroectoderm. Ttu-Zic expression in the earliest mesodermal cells may be consistent with that in vertebrates and ascidians (references in Merzdorf 2007). Most interestingly, the Ttu-Zic expression pattern in the segmented mesodermal cell lineage is very similar to the differentiation-stage-specific expression changes in Zic1, Zic2, and Zic3 that are observed in the course of mammalian somitogenesis (Inoue et al. 2007): Zic3 is detected in the earliest mesodermal cells adjacent to the primitive streaks, the Zic2 expression is enhanced in forming somites and most newly formed somites, and the expression of the three Zic genes continues in the dorsomedial part of the somite derivatives in later embryonic development.

Conservation of the two expression features between deuterostomes and protostomes suggests that a presumptive bilaterian ancestor had these expression features. It will be interesting to elucidate the Zic expression in other lophotrochozoans and to deduce the conserved expression features in this large taxon of Protostomia.

Acknowledgments We thank Takashi Inoue, Takahiko J. Fujimi, and other members of the Aruga laboratory for their helpful comments on the in situ hybridization experiments. This study was funded by the RIKEN Brain Science Institute.

\section{References}

Aruga J (2004) The role of Zic genes in neural development. Mol Cell Neurosci 26:205-221

Aruga J, Kamiya A, Takahashi H, Fujimi TJ, Shimizu Y, Ohkawa K, Yazawa S, Umesono Y, Noguchi H, Shimizu T, Saitou N, Mikoshiba K, Sakaki Y, Agata K, Toyoda A (2006) A wide-range phylogenetic analysis of Zic proteins: implications for correlations between protein structure conservation and body plan complexity. Genomics 87:783-792

Aruga J, Odaka YS, Kamiya A, Furuya H (2007) Dicyema Pax6 and Zic: tool-kit genes in a highly simplified bilaterian. BMC Evol Biol 7:201

Goto A, Kitamura K, Arai A, Shimizu T (1999a) Cell fate analysis of teloblasts in the Tubifex embryo by intracellular injection of HRP. Dev Growth Differ 41:703-713

Goto A, Kitamura K, Shimizu T (1999b) Cell lineage analysis of pattern formation in the Tubifex embryo. I. Segmentation in the mesoderm. Int J Dev Biol 43:317-327

Inoue T, Ota M, Mikoshiba K, Aruga J (2007) Zic2 and Zic3 synergistically control neurulation and segmentation of paraxial mesoderm in mouse embryo. Dev Biol 306:669-684 
Lichtneckert R, Reichert H (2005) Insights into the urbilaterian brain: conserved genetic patterning mechanisms in insect and vertebrate brain development. Heredity 94:465-477

Lindgens D, Holstein TW, Technau U (2004) Hyzic, the Hydra homolog of the zic/odd-paired gene, is involved in the early specification of the sensory nematocytes. Development 131:191-201

Matsuo K, Shimizu T (2006) Embryonic expression of a decapentaplegic gene in the oligochaete annelid Tubifex tubifex. Gene Expr Patterns 6:800-806

Merzdorf CS (2007) Emerging roles for zic genes in early development. Dev Dyn 236:922-940

Nakamoto A, Arai A, Shimizu T (2000) Cell lineage analysis of pattern formation in the Tubifex embryo. II. Segmentation in the ectoderm. Int J Dev Biol 44:797-805
Oyama A, Shimizu T (2007) Transient occurrence of vasa-expressing cells in nongenital segments during embryonic development in the oligochaete annelid Tubifex tubifex. Dev Genes Evol 217:675-690

Oyama A, Yoshida H, Shimizu T (2008) Embryonic expression of p68, a DEAD-box RNA helicase, in the oligochaete annelid Tubifex tubifex. Gene Expr Patterns 8:464-470

Shimizu T (1982) Development in the freshwater oligochaete Tubifex. In: Harrison FH, Cowden RR (eds) Developmental biology of freshwater invertebrates. Liss, New York, pp 283316

Takahashi H, Kamiya A, Ishiguro A, Suzuki AC, Saitou N, Toyoda A, Aruga J (2008) Conservation and diversification of Msx protein in metazoan evolution. Mol Biol Evol 25:69-82 